BNL -52248

DE90 014754
BNL- 52248

UC -414

(High Energy Physics-

DOE/OSTI-4500-R75)

\title{
SNAKES AND SPIN ROTATORS
}

S.Y. Lee

June 18, 1990

Accelerator Development Department

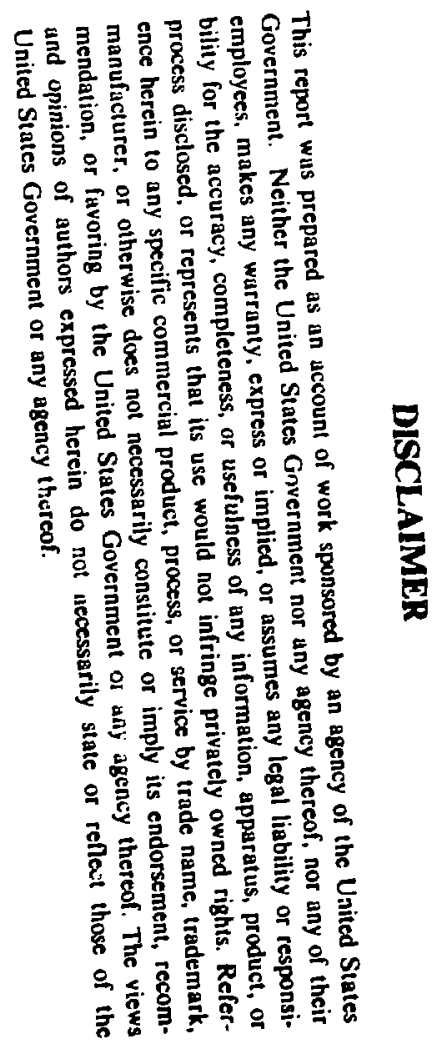

Brookhaven National Laboratory

Associated Universities, Inc.

Upton, Long Island, New York 11973

Under Contract No. DE-AC02-76CH00016 with the

UNITED STATES DEPARTMENT OF ENERGY

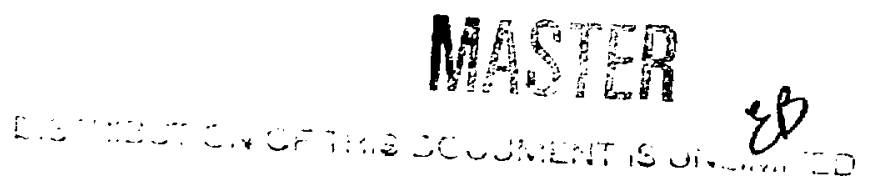




\begin{abstract}
The generalized snake configuration offers advantages of either shorter total snake length and smaller orbit displacement in the compact configuration or the multi-functions in the split configuration. We found that the compact configuration can save about $10 \%$ of the total length of a snake. On the other hand, the split snake configuration can be used both as a snake and as a spin rotator for the helicity state. Using the orbit compensation dipoles, the split snake configuration can be located at any distance on both sides of the interaction point of a collider provided that there is no net dipole rotation between two halves of the snake. The generalized configuration is then applite to the partial snake excitation. Simple formula have been obtained to understand the behavior of the partial snake. Similar principle can also be applied to the spin rotators. We also estimate the possible snake imperfections due to various construction errors of the dipole magnets. Accuracy of field error of better than $10^{-4}$ will be significant.
\end{abstract}




\section{Table of Contents}

$\begin{array}{ll}\text { Abstract } & \text { ii }\end{array}$

1. Introduction 1

2. Modified Snake Configuration 2

$2.1 \mathrm{~m}>2$ Case or the Compact Snake Configuration 3

$2.21<m<2$ Case 4

2.3 Bi-Sectional $\mathrm{m}=2$ Snake 5

3. Partial Snake 5

3.1 Compact Partial Snake 5

3.2 Split Partial Snake 6

$\begin{array}{ll}\text { 4. Snake Imperfections } & 7\end{array}$

$\begin{array}{llr}\text { 5. Conclusions } & 8\end{array}$

References $\quad 8$ 


\section{List of Figures}

Figure 1. The relation between $\psi_{x}, \psi_{y}$ of the spin rotation angle for the $H$ and $V$ magnets are shown. The snake axis $\phi_{\mathbf{s}}$ is also given as a function of $\phi_{y}$

Figure 2. Schematic plot of the modified snake configuration. The length of the horizontal bending magnet $\mathrm{mH}$ is determined by the closed orbit geometry.

Figure 3. The spin rotation angles $\psi_{\mathrm{x}}$ and $\psi_{\mathrm{y}}$ are plotted for $\mathrm{m}=1.106$, where the free space between the two halves of the snake is about $80 \mathrm{~m}$. The spin direction $\phi_{M}$ in the middle of the snake is also shown.

Figure 4. Schematic plot of the split snake configuration, where the distance between the two halves of the snake does not depend on the geometry. In this scheme, the aperture requirement for the dipole magnet is reduced in comparison with that of Fig. 2 with $m=1.106$.

Figure 5. The aperture requirement and the relation between $\psi_{x}$ and $\phi_{y}$ are plotted for the $5 \%$ compact snake configuration with $\mathrm{m}=2.42$. 


\section{List of Tables}

Table 1. Length and Orbit Displacements for Snake Configurations 4

Table 2. - Parameters for the Split Pàtial Snake 7 


\section{Iniroduction}

Recently, K. Steffen ${ }^{1}$ has discovered a families of snakes with the magnet sequence as

$$
S=(-H,-V, 2 H, 2 V,-2 H,-V, H)
$$

where $H$ and $V$ are the horizontal and vertical bending magnets respectively. To satisfy the snake criteria, the sequence of magnets does not alter the particle orbit outside the snake and the spinor of the particle is transformed according to

$$
e^{i \frac{\varphi}{2} \hat{n}_{a} \cdot \vec{\sigma}}
$$

where $\varphi$ is the spin rotation angle. At $\varphi=\pi$, we have a $100 \%$ snake. The snake axis $\hat{n}_{s}=\left(\cos \varphi_{s}, \sin \varphi_{s}, 0\right)$ depends on the excitation of $H$ and $V$ magnets. The Steffen's snake configuration $S$ can be summarized by the following equations

$$
\begin{aligned}
\cos ^{2} \psi_{y} & +\cos 2 \psi_{x} \sin ^{2} \psi_{y}=0 \\
\sin \varphi_{s} & =\sqrt{2} \cos \psi_{x}
\end{aligned}
$$

where $\psi_{x}$ and $\psi_{y}$ are the spin rotation angle of $H$ and $V$ magnets. The relation between $\psi_{x}$ and $\psi_{y}$ in Eq. (1) ensures the snake condition of the magnet sequence. The snake axis can then be determined by Eq. (2). The spin rotation angle $\psi_{y}$ is limited in the range between $45^{\circ}$ and $135^{\circ}$. Figure 1 shows the $\psi_{x}, \psi_{y}$ relationship of Eq. (1) and $\varphi_{s}$ vs. $\psi_{y}$.

The integrated magnet strength is given by

$$
\int B d \ell=1.746\left(6 \psi_{x}+4 \psi_{y}\right)[\mathrm{Tm}]
$$

Note that $\psi_{y} \in \pm\left[45^{\circ}, 135^{\circ}\right]$ and $\psi_{x} \in \pm\left[45^{\circ}, 90^{\circ}\right]$. The spin rotator axis $\varphi_{\text {s }}$ depends on the excitation of $\psi_{x}$ and $\psi_{y}$ is also shown in Fig. 1. When $\varphi_{s}=0$ or $180^{\circ}$, the snake axis is along the radial $\hat{x}$ axis. When $\varphi_{s}=90^{\circ}$, the snake axis is in the longitudinal direction. Depending on the excitation of the horizontal and vertical dipoles, the integrated magnet strengths vary from 17 to $33 \mathrm{Tm}$.

The corresponding orbit displacements are given by

$$
\begin{aligned}
& D_{x}=\left(\ell_{x}+\ell_{y}+2 \ell_{g}\right) \frac{\psi_{x}}{G \gamma} \\
& D_{y}=\left(2 \ell_{x}+\ell_{y}+2 \ell_{g}\right) \frac{\psi_{x}}{G \gamma}
\end{aligned}
$$


Since the maximum orbit displacement is proportional to the lengths of the magnets, it is advantageous to use a shorter dipole magnet. Since the lengths of magnets are given by

$$
\begin{aligned}
& \ell_{x}=1.746 \frac{\psi_{x}}{B[T]}[\mathrm{m}] \\
& \ell_{y}=1.746 \frac{\psi_{y}}{B[T]}[\mathrm{m}]
\end{aligned}
$$

Thus the length is inversely proportional to the constant magnetic field $B$ in Tesla. At RHIC injection energy, we expect $B \gamma \simeq 50 \mathrm{~T}$ with $B=2$ Tesla and $\gamma \simeq 26.6$. The orbit displacement can vary from $4 \mathrm{~cm}$ to $24 \mathrm{~cm}$ depending on the choice of magnet excitation. Economical consideration would prefer snake configurations with a smaller orbit distortion and smaller integrated $\int B d \ell$ of magnets.

The advantage of the Steffen snake configuration is that the snake axis $\vec{n}_{s}$ can be changed continuously by properly $\psi_{x}$ and $\psi_{y}$ excitation. However, this snake configuration suffers the rigid structure of magnet position. The minimum length requirement for the snake is given by

$$
L=6 \ell_{x}+4 \ell_{y}+6 \ell_{g}+\left[\ell_{x}+2 \ell_{g}\right]
$$

where $\ell_{x}, \ell_{y}$ and $\ell_{g}$ are respectively lengths of the magnets $H, V$ and the distance between adjacent magnets. The length of the snake configuration requires an extra space of $\ell_{x}+2 \ell_{s}$ in Eq. (3), which is a little bit too small to be useful and too large a space to be wasted.

In this paper, we shall address the modified magnet configuration for the snake. First it may be interesting to eliminate the wasted free space. Furthermore, it would be nice to divide the snake into two pieces, which can be fitted into two adjacent straight sections. To achieve these goals, we discuss the modified snake configurations and its applications in section 2. The idea is then applied to the partial snake in section 3 . the imperfection in the snake construction is discussed in section 4. Conclusion is given in section 5 .

\section{Modified Snake Configuration}

The essential feature of Steffen snake is the symmetric arrangement of vertical bending magnets and the anti-symmetric horizontal bending magnets. These features can be preserved by the following snake configuration

$$
S_{m}=(-H,-V, m H, 2 V,-m H,-V, H)
$$

where $m$ is a number determined by the geometry. When $m>2$, the space between the magnets $m H$ and $-m H$ can be minimized. When $1<m<2$, the snake can be 
decomposed into two parts, where each part is a spin rotator while the combined result of these two parts works as a snake. The spin rotation angle $\varphi$ and the snake axis angle $\varphi_{s}$ are given by

$$
\begin{aligned}
& \cos \frac{\varphi}{2}=\cos ^{2} \psi_{y}+\cos m \psi_{x} \sin ^{2} \psi_{y} \\
& \cos \varphi_{s}=\frac{-\sin \frac{m \psi_{x}}{2} \cos \psi_{y}}{\sqrt{\cos ^{2} \frac{m \psi_{x}}{2}+\sin ^{2} \frac{m \psi_{x}}{2} \cos ^{2} \psi_{y}}}
\end{aligned}
$$

Note here that the $m \psi_{x}$ vs. $\psi_{y}$ are the relevant variables in the equations to determine $\varphi$ and $\varphi_{s}$. Thus $\varphi_{s}$ is independent of $m$.

\section{1 $m>2$ Case or the Compact Snake Configuration}

The total length of the snake can be minimized by a proper chosen $m$. Assuming that the distance between adjacent magnets is $\ell_{g}$, the condition for zero orbit displacement outside the snake gives

$$
(m-1)\left(\ell_{y}+\ell_{g}+\frac{1}{2}(m-1) \ell_{x}\right)=\ell_{x}+\ell_{y}+2 \ell_{g},
$$

where $\ell_{x}, \ell_{y}$ are the 'engths of magnets $H$ and $V$ respectively. The total length of the snake configuration is given by

$$
L=6 \ell_{x}+4 \ell_{y}+6 \ell_{g}
$$

The snake property of the configuration is determined by Eqs. (9) and (10). The orbit displacements are given by

$$
\begin{aligned}
& D_{x}=\left(\ell_{x}+\ell_{y}+2 \ell_{g}\right) \frac{\psi_{x}}{G \gamma} \\
& D_{y}=\left(m \ell_{x}+\ell_{y}+2 \ell_{g}\right) \frac{\psi_{y}}{G \gamma}
\end{aligned}
$$

Table 1 compares the snake configuration for $\varphi_{s}=0^{\circ}, 45^{\circ}, 90^{\circ}, 135^{\circ}$ and $180^{\circ}$ snakes by assuming 2 Tesla magnetic field and $\ell_{g}=0.15 \mathrm{~m}$. Note that the compact snake configuration, where $m$ is obtained from Eq. (11), has a total length $1 \sim 2 \mathrm{~m}$ less than that of the Steffen snake configuration $(m=2)$. The horizontal orbit displacement is reduced slightly, while the vertical orbit displacement remains the same in both cases [see Eqs. (9), (10) and (14)]. The total integrated $\int B d \ell$ is also slightly smaller for the compact snake configuration. 
Table 1. Length and Orbit Displacements for Snake Configurations $\ell_{g}=0.15 \mathrm{~m}$.

\begin{tabular}{lllclll}
\hline $\mathrm{m}$ & $\psi_{x}$ & $\psi_{y}$ & $\phi_{s}$ & $\gamma D_{x}[\mathrm{~m}]$ & $\gamma D_{y}[\mathrm{~m}]$ & $\mathrm{L}[\mathrm{m}]$ \\
\hline 2 & 90 & 45 & 180 & 2.06 & 1.63 & 13.54 \\
2 & 60 & 54.74 & 135 & 1.13 & 1.58 & 10.94 \\
2 & 45 & 90 & 90 & 1.03 & 2.67 & 11.49 \\
2 & 60 & 125.26 & 45 & 1.82 & 4.92 & 15.24 \\
2 & 90 & 135 & 0 & 3.27 & 6.70 & 19.03 \\
2.334 & 77.12 & 45 & 180 & 1.62 & 1.63 & 11.48 \\
2.289 & 52.42 & 54.74 & 135 & 0.99 & 1.58 & 9.49 \\
2.209 & 40.74 & 90 & 90 & 0.91 & 2.67 & 10.37 \\
2.191 & 54.77 & 125.26 & 45 & 1.62 & 4.92 & 13.85 \\
2.215 & 81.26 & 135 & 0 & 2.84 & 6.70 & 17.09 \\
\hline
\end{tabular}

\section{$2.21<m<2$ Case}

When the snake is separated into two parts at the symmetry point, the combined effect on the spin remains unchanged provided that there is no net spin precession in the region between two half-snakes. To accomplish this task, $m$ can be determined from the geometry of Fig. 2, i.e.,

$$
(m-1)\left(d+\ell_{y}+\frac{1}{2}(m-1) \ell_{x}+\ell_{g}\right)=\ell_{x}+\ell_{y}+2 \ell_{g} .
$$

With the vertical compensation dipoles, $V^{\prime}$ and $-V^{\prime}$, the vertical orbit displacement in the central region will be zero.

The characteristics of the snake is governed by Eqs. (9) and (10). In the present scenario, the half-snake can be used either as a spin rotator or as a space saver, where the half-snake $\left(V^{\prime},-V^{\prime},-H,-V, m H, V\right)$ would occupy a straight section smaller than the full snake. To use the half-snake as a space saver, the accelerator lattice must possess adjacent straight sections. The horizontal orbital displacement is however slightly larger (see Eq. 13).

Using the half-snake as a spin rotator, besides its combined function as a snake, the helicity state of the spin particle can be achieved. For a spin up particle passing through the half snake, the spin components becomes

$$
\begin{aligned}
& S_{x}=-\sin m \psi_{x} \sin \psi_{y} \\
& S_{y}=\sin ^{2} \frac{m \psi_{x}}{2} \sin 2 \psi_{y} \\
& S_{z}=10
\end{aligned}
$$


Let $\phi_{M}$ be the angle of the spin relative to the radial $\hat{x}$ axis. We obtain then

$$
\tan \phi_{M}=\frac{S_{y}}{S_{x}}
$$

Figure 3 shows $\phi_{M}$ as a function of the $\psi_{y}$, the spin precession angle of the vertical bend magnet for $m=1.106$. The available free space is $80 \mathrm{~m}$ in the central region. Such a scheme can save four spin rotators in the helicity experiment. The horizontal orbit displacement for $m=1.1$ is about a factor of 2 larger than that of $m=2$. The orbit displacement problem in the central region can always be compensated by local orbit bumps without net spin precession angle.

Due to the large orbit displacement in the horizontal direction for the long spatial separation between the two half-snakes, the present scheme may be impractical for RHIC. The scheme is however useful for the snake to be used in the adjacent straight section separated by a quadrupole. A practical design of the combined snake and spin rotator will be discussed in the next section.

\subsection{Bi-Sectional $m=2$ Snake}

Since $m \simeq 1$ is needed to accommodate a large free space between the two halves of the snake, the orbit aperture becomes nearly a factor of 2 larger. The difficulty can be solved by the configuration of the snake shown in Fig. 4. Using the orbit compensation magnets $-H$ and $H$ is the middle of the two halves, where $m=2$ is restored. Similarly the $V^{\prime},-V^{\prime}$ magnet combination are used to correct the vertical orbit. In this scheme, the magnet excitation $\psi_{x}, \psi_{y}$ and the snake axis $\varphi_{\text {s }}$ are given by Fig. 1 discussed in the introduction. The spin direction in the free space is however rotated by an extra $\psi_{x}$ angle. The free space $2 \mathrm{~d}$ in Fig. 4 is adjustable provided that there is no residual dipole field in the straight section. The orbit displacement can be reduced by about a factor of 2 in comparison with that of Section 2.2.

\section{Partial Snakes}

\subsection{Compact Partial Snake}

The snake configuration discussed in section 2 can also be applied to the design of the partial snake. The total length of the partial snake can be minimized by a properly chosen $m$. Figure 5 (lower part) shows $\psi_{x}$ vs. $\psi_{y}$ for $5 \%$ snake. The total length of the $5 \%$ partial snake has a broad minimum of $2.556 \mathrm{~m}$ (with B $=2 \mathrm{~T}$ ) at $\psi_{y}=13.25^{\circ}$, while orbit displacements $D_{x}, D_{y}$ can be optimized at $\psi_{y} \simeq 10.5^{\circ}$. The length of the snake is 
about $2.6 \mathrm{~m}$ when 2 Tesla magneis and $15 \mathrm{~cm}$ spacing between magnets are sed. The corresponding $5 \%$ snake for $m=2$ will be about $50 \mathrm{~cm}$ longer.

To optimize the orbit displacements, we choose the following parameters for $5 \%$ snake at the peak field of $2 \mathrm{~T}$ with $15 \mathrm{~cm}$ space between magnets.

$$
\begin{aligned}
m & =2.45484 \\
\psi_{x} & =10.1359^{\circ} \quad\left(\ell_{x}=0.1544 \mathrm{~m}\right) \\
\psi_{y} & =10.5^{\circ} \quad\left(\ell_{y}=0.1600 \mathrm{~m}\right) \\
L_{\text {tot }} & =2.6072 \mathrm{~m}=8.5537 \mathrm{ft} \\
\gamma D_{x} & =6.06 \mathrm{~cm} \\
\gamma D_{y} & =8.58 \mathrm{~cm}
\end{aligned}
$$

At $1.4 \mathrm{~T}, 1.6 \mathrm{~T}$ and $1.8 \mathrm{~T}$ field strength, the snake reaches strengths of $2.47 \%, 3.22 \%$ and $4.06 \%$ respecivively.

Such a partial snake construction can be used in AGS, where the snake is needed at $G_{\gamma}>7$. The corresponding orbit displacements are less than $2 \mathrm{~cm}$. Since the orbit displacement is proportional to the magnet excitation strength, the orbital displacements in the $2.5 \%$ snake would be about $1.4 \mathrm{~cm}$ at $G \gamma=7$. The snake can be adiabatically turned on and off by a single power supply. The quadratic dependence of the magnet strength can be seen easily from the power series expansion of Eq. (9), i.e.

$$
\varphi \simeq 2 m \psi_{x} \psi_{y}
$$

Eq. (16) indicates that the snake strength $\varphi$ depends on $\psi_{x}$ and $\psi_{y}$ in the bilinear form (see also the lower part of Fig. 5 for $\varphi=9^{\circ}$ ). Similarly, the snake axis is given by

$$
\varphi_{s} \simeq \frac{\pi}{2}+\frac{m \psi_{x}}{2}
$$

Therefore all the partial snakes with small $\psi_{x}$ and $\psi_{y}$ to obtain minimum orbit displacement and total length has snake axis near the longitudinal direction. The type II snake, where $\varphi_{s}=0$ or $180^{\circ}$ requires large $\psi_{x}$. This characteristic feature appears in all kinds of snake configurations.

\subsection{Split Partial Snake}

For the low energy accelerator, a long free space is usually not easily accessible. However two straight sections separated by a quadrupole can be useful for the partial snake as 
well. The length of the quadrupole determines the quantity $m$. Two halves of a snake can be optimized to occupy the available space. In Table 2 we list the snake configuration for $d=0.45 \mathrm{~m}$ and $B=2 \mathrm{~T}$, where the corresponding free space is $90 \mathrm{~cm}$.

Table 2. Parameters for the Split Partial Snake.

\begin{tabular}{lccccccc}
\hline \% Snake & $\mathrm{m}$ & $\ell_{x}[\mathrm{~m}]$ & $\ell_{y}[\mathrm{~m}]$ & $\ell_{y}^{\prime}[\mathrm{m}]$ & $\gamma D_{x}(\mathrm{~cm})$ & $\gamma D_{y}(\mathrm{~cm})$ & $L_{1 / 2}[\mathrm{~m}]$ \\
\hline 5 & 1.7903 & 0.2021 & 0.1676 & 0.3053 & 8.65 & 8.88 & 2.250 \\
10 & 1.8585 & 0.2894 & 0.2286 & 0.4244 & 15.12 & 15.57 & 2.883 \\
\hline
\end{tabular}

The $\ell_{x}, \ell_{y}, \ell_{y}^{\prime}$ are the lengths of magnets $H, V$ and $V^{\prime}$ shown in Fig. 2 respectively. $D_{x}$ and $D_{y}$ are the maximum the orbit displacements in the horizontal and vertical planes. $L_{1 / 2}$ is the total length of the half-snake including the vertical orbit correctors $V^{\prime}$ and $-V^{\prime}$. Since the orbit displacements vary inversely with respective to the energy and vary proportional to the magnet excitation strength, the orbit displacement can be maintained to be less than $1 \mathrm{~cm}$ in the realistic application.

\section{Snake Imperfections}

Since the snakes are composed of transverse magnets, there are two possible random errors: dipole excitation and dipole rotation. These two types of errors can give rise to error in the spin precession argle $\Delta \varphi$ and in the snake axis $\Delta \varphi_{s}$ of the order

$$
\Delta \varphi_{s} \sim \Delta \varphi \sim \sqrt{\left(m \Delta \psi_{x}\right)^{2}+\left(\Delta \psi_{y}\right)^{2}+\left(\Delta \theta_{R}\right)^{2}}
$$

where $\Delta \psi_{x}, \Delta \psi_{y}$ and $\Delta \theta_{R}$ are rms errors in the $H, V$ magnets and dipole rotation respectively. These errors should be in the order of $10^{-3}$, which give rise to the same order of magnitude of errors in $\Delta \varphi_{3}$ and $\Delta \varphi$. The tolerance of the error discussed in ref. 2 is much larger. Besides the random error, the dependent of $\varphi$ and $\varphi_{s}$ on the systematic error can be obtained by

$$
\begin{aligned}
\Delta \varphi & =\left[\left(\frac{\partial \varphi}{\partial \psi_{x}}\right)^{2}\left(\Delta \psi_{x}\right)^{2}+\left(\frac{\partial \varphi}{\partial \psi_{y}}\right)^{2}\left(\Delta \psi_{y}\right)^{2}\right]^{1 / 2} \\
\Delta \varphi_{s} & =\left[\left(\frac{\partial \varphi_{s}}{\partial \psi_{x}}\right)^{2}\left(\Delta \psi_{x}\right)^{2}+\left(\frac{\partial \varphi_{s}}{\partial \psi_{y}}\right)^{2}\left(\Delta \psi_{y}\right)^{2}\right]^{1 / 2}
\end{aligned}
$$

Figure 1 shows clearly that the snake axis $\varphi_{s}$ is much more sensitive to $\psi_{y}$ for the type II snakes, where $\varphi=0$ or $\pi$. 
We obtain from Eqs. $(9,10)$ that

$$
\begin{aligned}
\Delta \varphi & \simeq \pm 2\left[\cot \frac{m \psi_{x}}{2} \Delta\left(m \psi_{x}\right) \pm 2 \cot \psi_{y} \Delta \psi_{y}\right] \\
\Delta \varphi_{\mathrm{s}} & =\left( \pm \Delta \psi_{y}-\frac{\sqrt{2}}{2} \cos \frac{m \varphi_{x}}{2} \cos \psi_{y} \Delta\left(m \psi_{x}\right)\right) / \sin \varphi_{\mathrm{s}}
\end{aligned}
$$

Due to $\sin \varphi$, in the denominator, the type II snake is much harder to maintain its accuracy in the snake axis. It is preferable to tune the snake away from the $\varphi_{0}=0$ or $\pi$.

Finally for the split configuration discussed in Section 2.3, the accuracy in the orbit compensation magnets $-H$ and $H$ is also important. The snake axis will deviate from the horizontal plane by an angle equal to the net spin rotation angle of $-H$ and $H$ magnets.

The antisymmetric insertion in the RHIC and SSC can also give rise to a net spin rotation for large amplitude particles. Using the RHIC lattice function, we expect a net spin rotation angle of $6 \times 10^{-2}$ radian for the largest amplitude particle (35 mrn from the center of the quadrupole) at the top energy. The effect decreases with increasing $\beta^{*}$ value at the collision point. Since the large amplitude particle executes betatron motion, the snake axis will oscillate around the desired value with betatron frequency. Small amplitude oscillation of the spin tune around $1 / 2$ will be an interesting problem to be studied. To minimize the effect, we can decrease the betatron amplitude at the high $\beta$ quadrupoles. However the luminosity will be correspondingly smaller.

\section{Conclusions}

The generalized snake configuration has been found to be useful in realistic applications for either compact or split geometries. The basic properties of these snakes configurations has been studied. We found that the split snake can be used as a snake as well as the spin rotator for the helicity state. The generalized snake structure can be applied to a partial snake, which can be optimized to minimize orbit displacements and the total length of the magnets. Such an optimization is usually needed for the small accelerator, where the length of the straight section is small. Error anaiysis of the snake is also discussed.

\section{References}

1. K. Steffen, Particle Accelerators, 24, 45 (1989).

2. S.Y. Lee and E.D. Courant, Phys. Rev. D41, 292 (1990). 


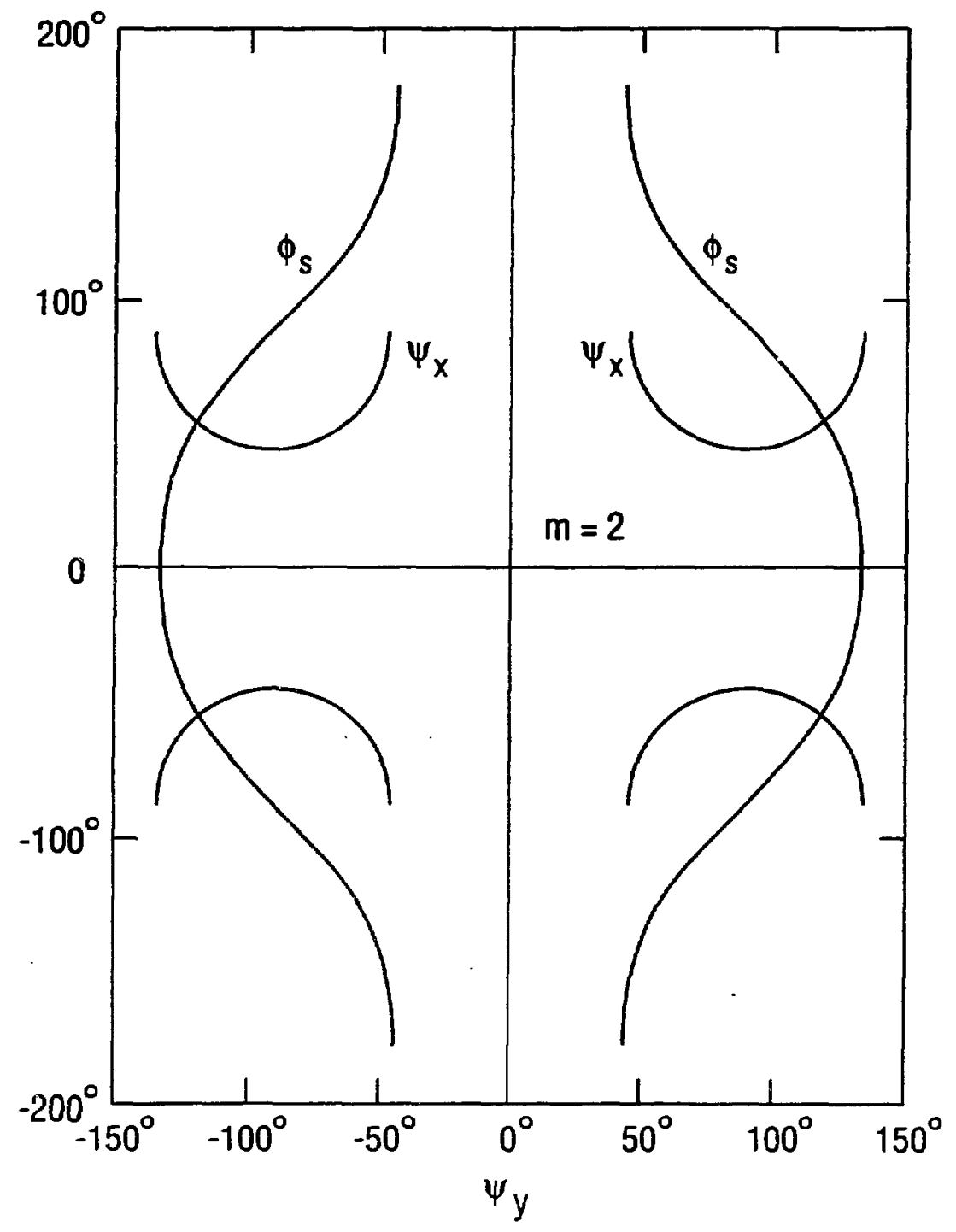

Figure 1. 


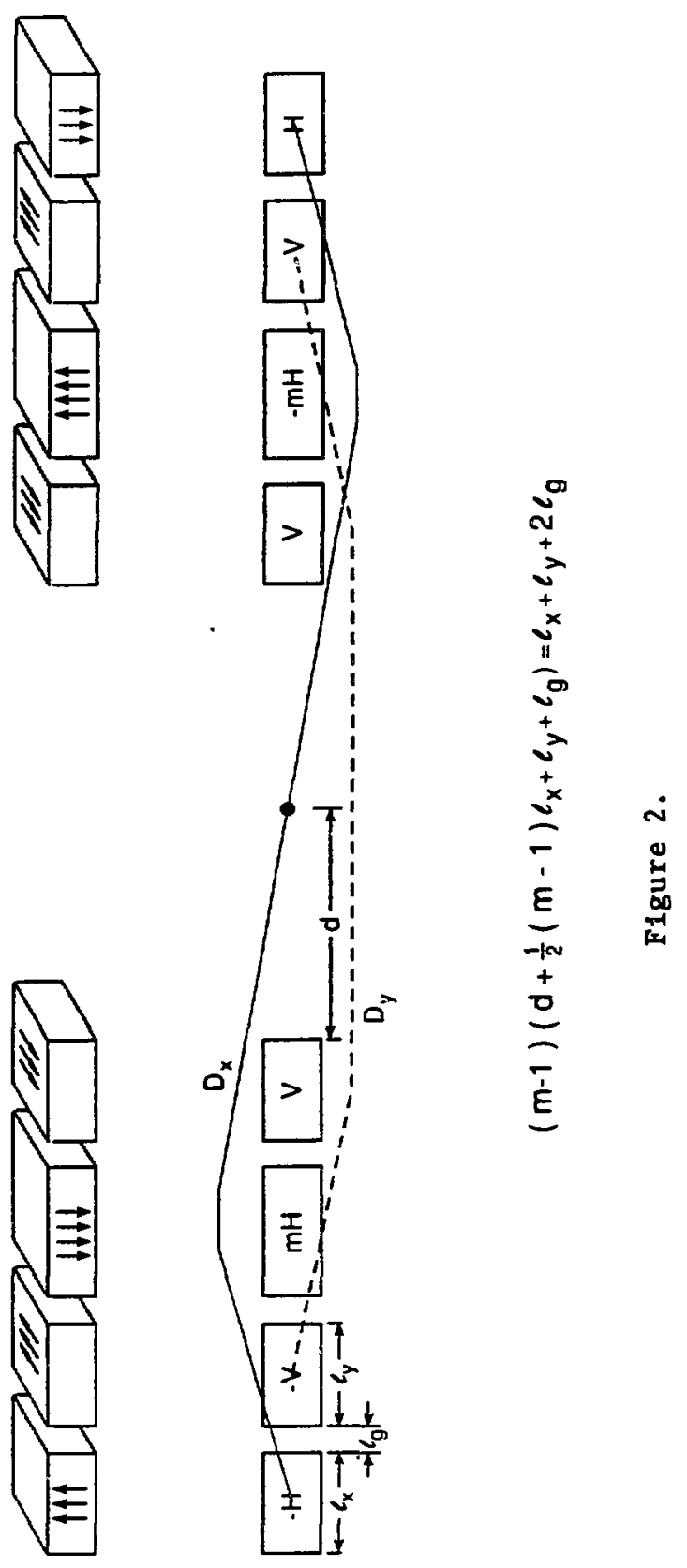




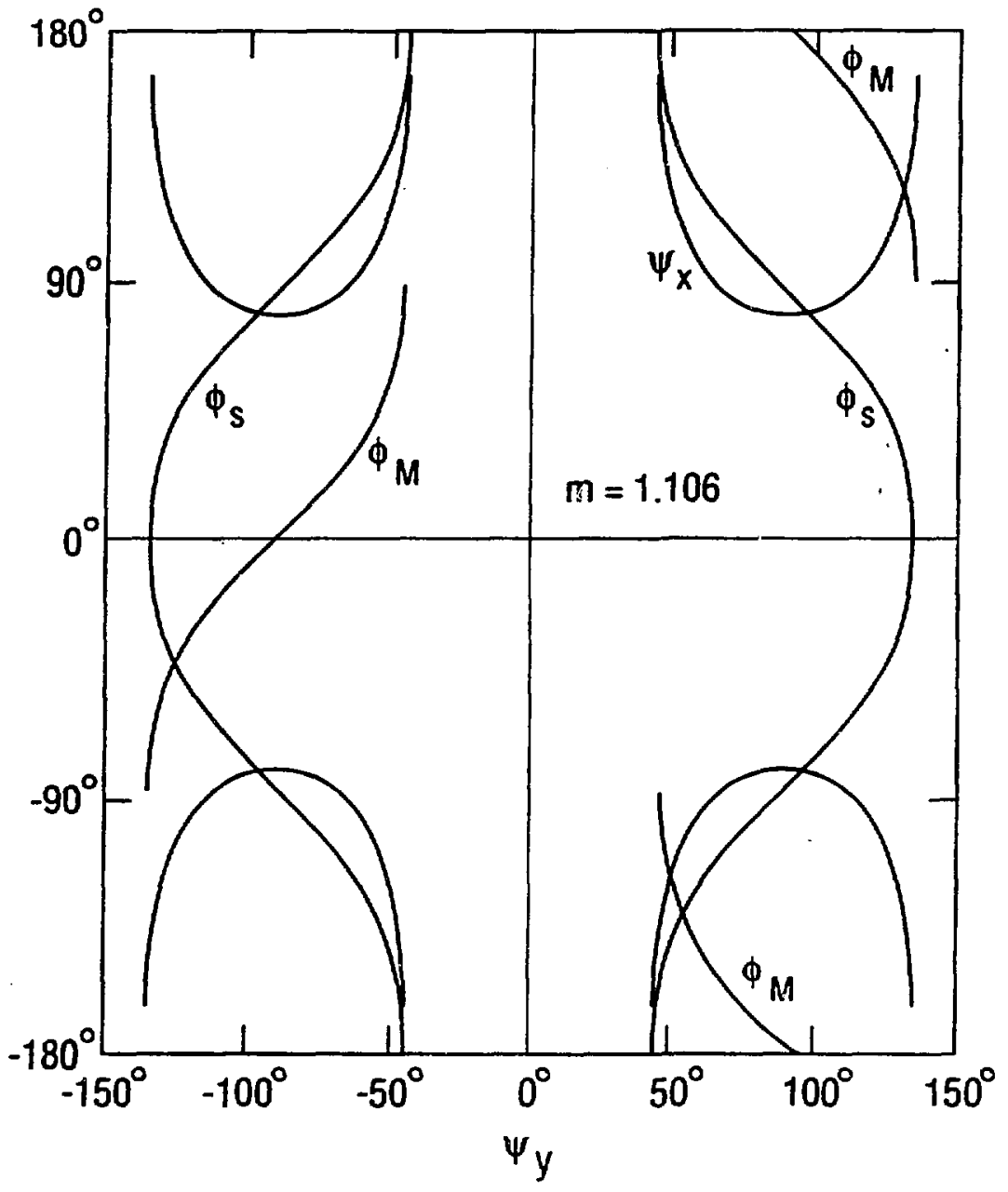

Figure 3. 


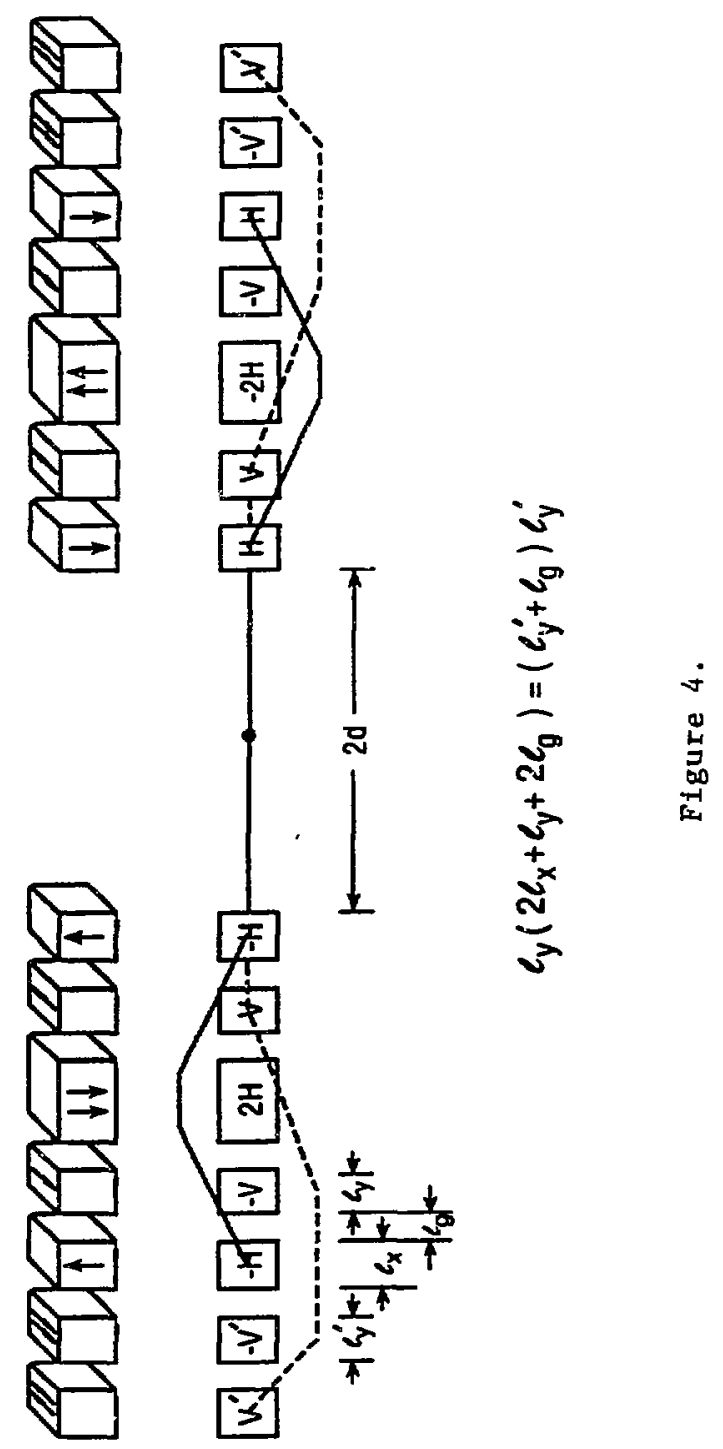




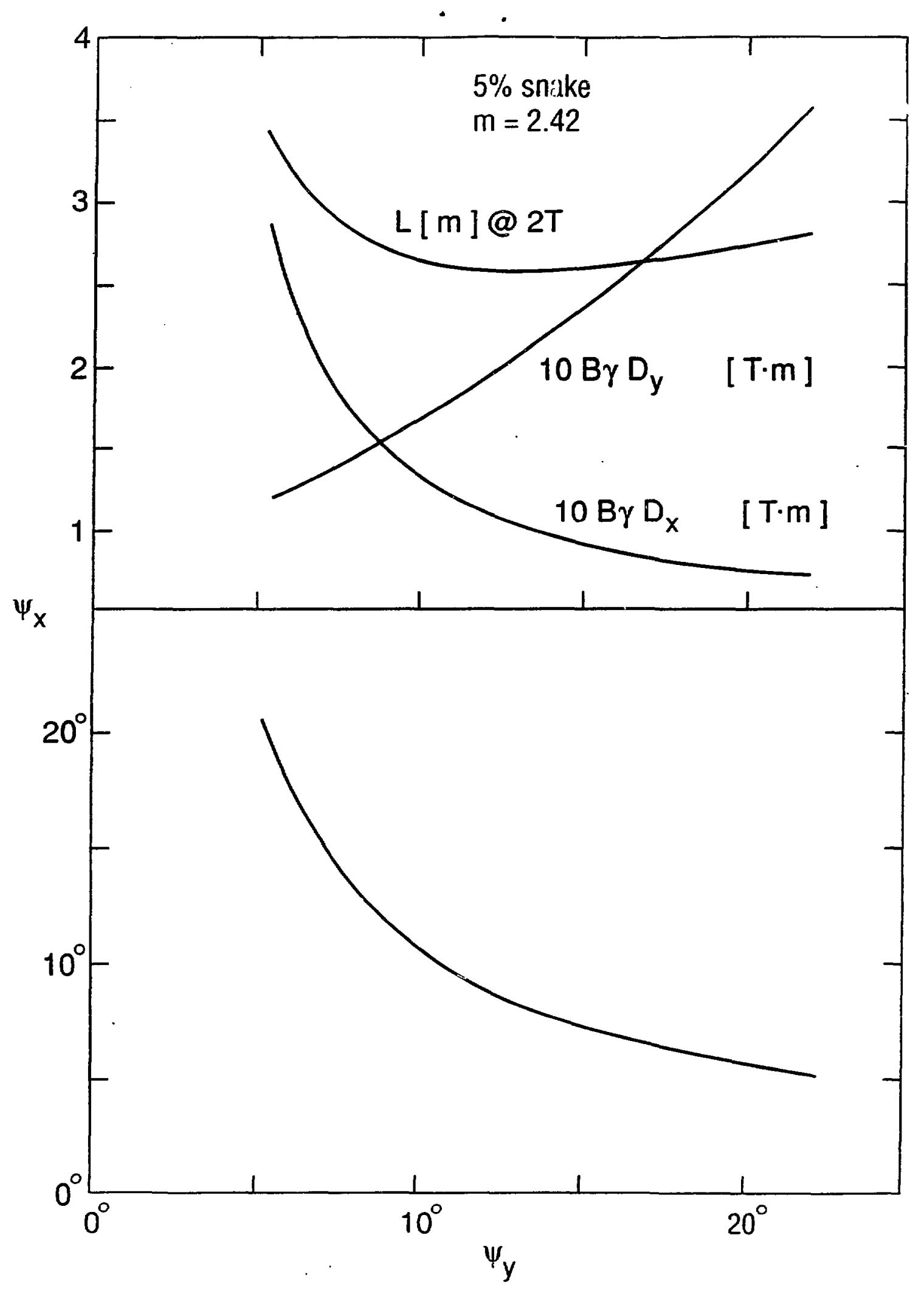

Figure 5 . 